framework for long term conditions in order to improve the service delivery to children with epilepsy.

Method A questionnaire was developed using CG137epilepsy NICE guideline. Patients with epilepsy who were seen over the period of January-May 2014 were selected to receive the questionnaires that included parents feeling once the diagnosis is made. Patients were seen both in hospital and community settings.

Results 40 received the questionnaires, 34 returned (85\%). Most (30/34) were younger than 10 years at the time of diagnosis. Co-morbidities were seen in $62 \%$ including cerebral palsy, learning difficulty, autism, ADHD and some had combinations. The high number relates to seeing more children in special schools over the period of the audit. 18/40 reported significant emotional problems at diagnosis: sad, terrified, devastated and shocked. Information on lifestyle including water safety was provided to $25 / 34$ (70\%). Information on SUDEP was not provided to $23 / 34(68 \%)$.

Conclusion There is a wide variation in practice within the Department. There is no epilepsy clinic with no epilepsy nurse. The information provided to patients was very patchy, too little or given too late.

Action Proforma was put in place, covered classification as per Multi-axial diagnostic scheme: Description of seizures, Seizure type, Syndrome, Aetiology and Co-morbidity. Referral letter is sent to the community nursing team for early home visit and an early liaison with school to improve outcome. Group training for the parents was commenced.

\section{G200(P) A CASE HIGHLIGHTING THE IMPACT OF UNCORRECTED SCOLIOSIS}

S Sanapala. Southmead Hospital, North Bristol NHS Trust, Bristol, UK

10.1136/archdischild-2015-308599.194

Method This is a case of a previously well 17 year old girl with cerebral palsy secondary to Lissencephaly. She presented with a history of intractable vomiting progressing over the course of a few months secondary to severe worsening scoliosis.

Upon further investigation, it was felt that the extensive degree of her scoliosis was rotating her abdominal anatomy in such a manner to be the main cause of her vomiting. She was reviewed by the orthopaedic team who felt that correction of her scoliosis was indicated however this was not without significant risks to the patient especially given her extensive recent weight loss secondary to the vomiting. However, without the procedure the patient would continue to vomit and losing weight and may eventually die.

Result The parents of the patient were keen to proceed with the operation despite the high risks as they felt their daughter's quality of life had deteriorated significantly since the onset of the scoliosis and recurrent vomiting.

The patient underwent the scoliosis correction and made a good recovery.

Conclusion This case presents a dilemma in which without correction of her scoliosis, the patient would continue to vomit and lose weight (medical therapy had failed). However, undertaking such a major operation in this patient carried a significant mortality risk.

The case also highlights the importance of close follow up of these patients as this child did not develop scoliosis until her teenage years and she missed her follow up appointments due to an address change.

Pictures of the scoliosis (X ray/CT are available to accompany the case)

\section{G201(P) DO BASELINE BLOOD PRESSURE AND HEART RATE IN CHILDREN WITH A SPINAL CORD INJURY VARY DEPENDING ON THEIR LEVEL OF INJURY?}

${ }^{1} \mathrm{E}$ Gustafsson Oberink, 'S Gale, ${ }^{2} \mathrm{~A}$ Graham. National Spinal Injury Unit, Stoke Mandeville Hospital, Aylesbury, UK

\subsection{6/archdischild-2015-308599.195}

Children with a spinal cord injury may have blood pressure and heart rate differences from the expected values of that age. Understanding this potential problem is important for optimal management.

Aims To establish any association between level of spinal injury and ASIA (American Spinal Injury Association) impairment scale severity score with heart rate, blood pressure and diurnal variation in blood pressure. The association between age, gender and BMI on heart rate and blood pressure was also explored.

Method Retrospective records were reviewed for 32 children admitted to a National Spinal Injury Centre for more than 4 rehabilitation periods between 2011 and 2013 (107 admissions). Patient and injury data were established. The first recorded morning and evening blood pressures and heart rates were collated.

Data was analysed using MS Excel 2010 and IBM SPSS v.20. Results The mean age at first admission was 10.2 years, $41 \%$ were male, and $59 \%$ had an injury at or above T6. Only $12 \%$ of the admissions had complete cardiovascular data.

The associations between ASIA score and BP centile group; ASIA score and diurnal variation; level of injury and BP centile group; and level of injury and diurnal variation, were not statistically significant.

Increasing age was significantly associated with a lower heart rate (OR 0.094, p value <0.001) and systolic BP with increasing BMI (OR 2.97, 95\% CI(1.439, 6.137)

Conclusion The changes observed with age and BMI can be accepted as normal physiological change. That no statistical association between the injury related factors, ASIA score and level of injury, and cardiovascular measures was observed could be due to the poor data quality, and no conclusion can therefore be reached from this finding.

Improved measurement and recording of height, weight and cardiovascular observations is paramount for optimal cardiovascular management in this patient group.

\section{G202(P) SLEEP MANAGEMENT IN AUTISTIC SPECTRUM DISORDER}

${ }^{1} S$ Jmor, ${ }^{2} E$ Abbas. 'Medical School, Birmingham University, Birmingham, UK; ${ }^{2}$ Community Child Health, Wrightington Wigan and Leigh NHS FT, Wigan, UK

\subsection{6/archdischild-2015-308599.196}

Abnormal sleep affects 44-89\% of children with ASD. Currently there are no official UK guidelines for the management of sleep with ASD.

Aim To discuss sleep management using a case study of 9 year old boy with ASD. 